\title{
High ICAM-1 gene expression in pulmonary fibroblasts of COPD patients: a reflection of an enhanced immunological function
}

\author{
A. Zandvoort*,\#, Y.M. van der Geld*, M.R. Jonker*,\#, J.A. Noordhoek* , J.T.W.M. Vos*, \\ J. Wesseling*, H.F. Kauffman ${ }^{\Uparrow}$, W. Timens* and D.S. Postma ${ }^{\#}$
}

ABSTRACT: Chronic obstructive pulmonary disease (COPD) is characterised by destruction of extracellular matrix (ECM) in parenchymal areas, whereas the bronchial walls can show fibrosis. In addition, an extensive inflammatory process is observed. CD8+ T-cells, located throughout the lung, and epithelial cells in centrally located airways, produce cytokines involved in the inflammatory process. These cytokines may influence the present fibroblasts, the key effectors in the defective ECM repair and maintenance in COPD.

The current authors explored the effects of the cytokine microenvironment on cell-cell interaction gene expression in pulmonary fibroblasts of controls $(n=6)$, and Global Initiative for Chronic Obstructive Lung Disease stage II $(n=7)$ and stage IV $(n=7)$ COPD patients. The current authors simulated the in vivo microenvironment using supernatants of CD3/CD28 stimulated CD8+ T-cells isolated from peripheral blood of COPD patients, supernatant of a bronchial-epithelial cell line, or a combination of both.

The present data show that fibroblasts of chronic obstructive pulmonary disease patients display an altered response to the cytokine microenvironment, depending on both the disease stage and the central or peripheral location in the lung. Especially adhesion-related genes are upregulated in fibroblasts of chronic obstructive pulmonary disease patients, which can indicate a more pronounced role of fibroblasts in the inflammatory process in chronic obstructive pulmonary disease, possibly resulting in reduced function as effectors of extracellular matrix repair.

KEYWORDS: CD8+ T-cells, chronic inflammation, chronic obstructive pulmonary disease, intracellular adhesion molecule-1, lung fibroblasts

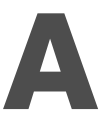
pproximately $15-20 \%$ of cigarette smokers develop chronic obstructive pulmonary disease (COPD), which comprises a heterogeneous combination of chronic bronchitis and emphysema [1, 2]. Emphysema is characterised by chronic inflammation, alveolar destruction and loss of alveolar attachments, causing a permanent enlargement of airspaces and loss of elastic recoil of peribronchiolar attachments, which results in airflow limitation $[1,3,4]$. To date, the underlying mechanisms of the vulnerability to cigarette smoke have not been elucidated $[1,2,5,6]$.

The present authors proposed that besides the generally accepted imbalance between oxidants and antioxidants and between proteases and their inhibitors, resulting in excess tissue destruction, a hampered tissue repair process is involved in the pathogenesis of emphysema $[7,8]$.
Fibroblasts are important in tissue repair and modulation by producing extracellular matrix (ECM) proteins, ECM-degrading enzymes and their inhibitors [9]. The current authors have previously observed that lung fibroblasts of patients with severe COPD exhibit an altered ECM production profile compared with fibroblasts of controls pointing to an important role of fibroblasts in the defective ECM modulation observed in COPD [8, 10-12].

Besides tissue degradation and destruction, a chronic inflammatory process underlies COPD, with infiltrating inflammatory cells producing a large variety of cytokines [3, 6, 13-16]. In addition, cigarette smoke damages epithelial cells, cells that are an important source of growth factors, e.g. transforming growth factor (TGF)- $\beta$ [3]. This complex cytokine microenvironment most probably contributes to the altered
AFFILIATIONS

Depts of * Pathology and Laboratory Medicine,

\#Pulmonology and

"Allergology, University Medical Center Groningen, University of Groningen, The Netherlands.

CORRESPONDENCE

W. Timens

University Medical Center Groningen Dept of Pathology and Laboratory

Medicine

Hanzeplein 1

9713 GZ Groningen

The Netherlands

Fax: 31503632510

E-mail: w.timens@path.umcg.nl

Received:

October 052005

Accepted after revision:

March 212006

SUPPORT STATEMENT

The present study was supported by a grant from the Netherlands Asthma

Foundation (NAF 99.15) 
behaviour of pulmonary fibroblasts of COPD patients. In addition to ECM production, fibroblasts can be actively involved in the inflammatory processes via cytokine and chemokine production and by direct interaction with inflammatory cells [7, $17,18]$. The present authors hypothesise that the local cytokine microenvironment determines the balance between ECM production and pro-inflammatory functions of fibroblasts.

The current study was conducted to further explore the role of fibroblasts in COPD and especially to investigate the influence of the complex cytokine microenvironment of the diseased lung on cell-to-cell interaction and matrix production. As in vivo analysis of human pulmonary fibroblasts is not possible, the current authors designed an in vitro model of the local cytokine microenvironment of the diseased lung, using supernatants of stimulated epithelial and CD8+ T-cells. The cytokine microenvironment is complex and consists of many cytokines and chemokines. By using supernatants a model was generated that was as close as possible to the in vivo situation. Epithelial and CD8+ T-cells are known as key sources of the cytokines in the fibroblast microenvironment of the diseased lung [19]. Therefore, fibroblasts were treated either with a supernatant of CD3/CD28 stimulated CD8+ T-cells isolated from peripheral blood of COPD patients, or a supernatant of a bronchial epithelial cell line or a combination of both supernatants. The effects of the supernatants on the expression of cell-interaction and matrix related genes in fibroblasts by real time PCR, using CDNA- macro-filter array as initial gene expression exploration, were compared. Protein expression analysis was used to confirm mRNA expression. Fibroblasts from both COPD patients (Global Initiative for Chronic Obstructive Lung Disease (GOLD) stage II/III and stage IV) and control patients were studied.

\section{MATERIALS AND METHODS \\ Subjects}

To investigate mRNA expression, lung tissue was obtained from 20 individuals. The clinical characteristics of the groups are presented in table 1 . Informed consent was given by all patients. Classification of severity of COPD was based on the 2003 GOLD criteria [4, 20]. Three groups were studied: 1) GOLD stage II/III $(n=7)$; this group included individuals with moderate (GOLD stage II, $\mathrm{n}=5$ ) and severe COPD (GOLD stage III, $n=2)$. 2) GOLD stage IV $(n=7)$; this group contained individuals with very severe COPD. 3 ) Control $(n=6)$; this group included individuals with histologically normal lungs. Patients had no $\alpha_{1}$-antitrypsin deficiency. Emphysema was defined as histologically abnormal enlargement of the airspaces distal to the terminal bronchioles, accompanied by destruction of alveolar walls and without obvious fibrosis $[1,2$, 4], and was assessed by routine histological examination of lung tissue by an experienced pulmonary pathologist.

GOLD stage II/III tissue was obtained from noninvolved lung tissue from patients undergoing resective surgery for pulmonary carcinoma. Tissue was always taken as far away as possible from the tumour, or from a noninvolved lobe. Histopathologically, the emphysematous lesions were present, yet lesions were of limited but varying severity. Moderate emphysema was noted histopathologically as isolated or unattached segments of viable alveolar septal tissue or isolated crosssections of pulmonary vessels.

\begin{tabular}{|c|c|c|c|}
\hline \multirow{2}{*}{ TABLE 1} & \multicolumn{3}{|c|}{ Characteristics of the patient and control groups } \\
\hline & Control & GOLD stage II/III & GOLD stage IV \\
\hline Subjects & 6 & 7 & 7 \\
\hline Age yrs & $44.5(25-71)$ & $69(44-81)$ & $55(44-61)$ \\
\hline \multicolumn{4}{|l|}{ Sex } \\
\hline Male & 3 & 7 & 1 \\
\hline Female & 3 & 0 & 6 \\
\hline \multicolumn{4}{|l|}{ Smoker } \\
\hline Ex-smoker ${ }^{\#}$ & ND & 2 & 7 \\
\hline Current & ND & 5 & 0 \\
\hline Pack-yrs & ND & $40(14-58)$ & $30(12-54)$ \\
\hline FEV $1 \%$ pred & ND & $73(40-76)$ & $18(13-29)$ \\
\hline FEV1/FVC \% & ND & $55(44-60)$ & $28(24-66)$ \\
\hline
\end{tabular}

Data are presented as n or median (range). GOLD: Global Initiative for Chronic Obstructive Lung Disease; FEV1: forced expiratory volume in one second; \% pred: percentage predicted; FVC: forced vital capacity; ND: not determined. \# Ex-smokers had not smoked for a minimum of $1 \mathrm{yr}$.

Tissue from the very severe COPD group (GOLD stage IV) was obtained from patients with COPD undergoing surgery for lung transplantation or lung volume reduction. The resected tissue showed both macroscopically and microscopically severe emphysematous lesions, often accompanied by bullae.

The control group consisted of donor lung tissue that had not been used for transplantation because of technical or logistical (unilateral transplantation) reasons. Technical reasons were related to doubt as to technical transplantability in relation to available recipients or doubts concerning the condition of the lung. In case of the latter, lung tissue was included only after additional investigations (including histopathology), and when it showed no signs of COPD [1], or of any other significant pathology.

\section{Isolation and culture of lung fibroblasts}

Pulmonary fibroblast cultures were established from parenchymal lung tissue by means of an explant technique as described previously [11]. Absence of mycoplasma contamination in the fibroblast cultures was confirmed with a mycoplasma detection kit (Roche Diagnostics, Almere, the Netherlands). Isolated cells were characterised as fibroblasts by morphological appearance and expression pattern of specific proteins [11]. All cells exhibited a characteristic staining pattern for vimentin, fibronectin, and the fibroblast marker prolyl-4-hydroxylase and lacked immunoreactivity for keratin. It was found that $<5 \%$ of cells were positive for desmin and $\alpha$-smooth muscle cell actin.

\section{Supernatants}

Epithelial supernatant was obtained from a bronchial epithelial cell line, 16HBE [21] (kind gift from D.C. Gruenert, University of Vermont, California Pacific Medical Center Research Institute, San Francisco, CA, USA). The cell line 16HBE was grown to confluence in complete medium (Ham's F12 medium; BioWhittaker Cambrex, Verviers, Belgium) supplemented with $10 \%$ foetal calf serum (FCS; PAA laboratories, Linz, Austria), $2 \mathrm{mM} \mathrm{L-glutamine,} 100 \mu \mathrm{g} \cdot \mathrm{L}^{-1}$ streptomycin and 
$100 \mathrm{U} \cdot \mathrm{mL}^{-1}$ penicillin (BioWhittaker Cambrex). After confluence, culture medium was replaced with medium containing $0.5 \%$ FCS for $24 \mathrm{~h}$. This supernatant was harvested and stored at $-80^{\circ} \mathrm{C}$

To obtain culture supernatant of CD8+ T-cells, peripheral blood was collected from seven patients with COPD. Informed consent was given by all patients. Heparinised blood was obtained and layered on Ficoll-paque (Amersham Biosciences, Uppsala, Sweden). The interface containing peripheral blood mononuclear cells (PBMCs) was collected. PBMCs were resuspended at $1 \times 10^{7}$ cells $\cdot \mathrm{mL}^{-1}$ in Hank's balanced salt solution $/ 2 \%$ FCS for purification of CD8 cells. CD8 cells were isolated by positive selection using anti-CD8 magnetic beads and Detach-a-bead (Dynal, Wirral, UK). The purity of the isolated CD8 T-cells was determined by fluorescence-activated cell sorter analysis on a coulter Epics ELITE (Beckman Coulter, Hialeah, FL, USA) with CyQ-labelled anti-CD8, phycoerythrin labelled anti-CD4 and fluorescein isothiocyanate-labelled antiCD3 antibodies (BD Bioscience, Oxford, UK). The CD8 cells were $87 \%$ pure containing $7 \%$ CD4 T-cells. Isolated CD8 cells were resuspended in culture medium containing $0.5 \%$ FCS and incubated for $24 \mathrm{~h}$. Isolated CD8 cells were stimulated with anti-CD3 and anti-CD28 antibodies by replacing the culture medium with $0.5 \%$ FCS medium containing 5\% anti-CD3 (clone WT-32) and anti-CD28 antibodies (clone 20-4996; CLB Sanquin, Amsterdam, the Netherlands) for $24 \mathrm{~h}$. Supernatant was then harvested, centrifuged and stored at $-80^{\circ} \mathrm{C}$.

\section{Fibroblast culture for cDNA arrays}

Fibroblasts from confluent cultures were seeded in culture plates (Corning BV, Amsterdam, the Netherlands) in complete medium and grown to confluence (passage 5). Culture medium was replaced with medium containing $0.5 \%$ FCS for $24 \mathrm{~h}$. Medium was replaced with new $0.5 \%$ FCS medium, either with or without supernatant of the 16HBE cell line (1 in 25 dilution), supernatant of CD8 cells stimulated with anti-CD3 and anti-CD28 ( 1 in 10 dilution) or a combination of supernatant of 16HBE cells and CD8 cells ( 1 in 25 and 1 in 10 final dilution concentration, respectively). Optimal stimulation concentration and duration was determined in pilot experiments (data not shown). After $6 \mathrm{~h}$ cells were harvested for RNA isolation.

\section{Preparation of RNA and hybridisation of cDNA arrays}

Fibroblast total RNA was isolated using the RNeasy mini kit (Qiagen, Hilden, Germany). RNA quantity was determined by optical density measurements at $260 \mathrm{~nm}$. A total of $3 \mu \mathrm{g}$ RNA per patient was pooled per group. Total RNA was treated with DNase I and run over a column to remove genomic DNA (Qiagen).

The Atlas Cell Interaction expression array (BD Biosciences Clontech, Palo Alto, CA, USA) was used to identify differences in the expression pattern of 265 known genes in the fibroblasts from the three patient groups treated with supernatants of $16 \mathrm{HBE}$ cells, CD8 cells, or their combination. The Atlas expression arrays are based on a filter array technique (macro-array) that uses a nylon filter spotted with cDNAs of the genes of interest that can be hybridised with cDNA of the samples to be analysed. A total of $2-5 \mu \mathrm{g}$ total RNA was used for cDNA synthesis using a commercial kit (BD Biosciences).
The cDNA was labelled by incorporation of $\left[\alpha_{-}{ }^{32} \mathrm{P}\right]$ deoxycytidine triphosphate $\left(10 \mu \mathrm{Ci} \cdot \mu \mathrm{L}^{-1}\right)$. The probes were hybridised when the counts $\min ^{-1}$ were $2-8 \times 10^{6}$, with the ExpressHyb hybridisation solutions applied to the cDNA arrays following the user manual (BD Biosciences). The membranes were exposed to a phosphorimaging screen at room temperature for 3 days. Quantification of bound cDNA was performed using Phosphorimaging system (Molecular Dynamics, Sunnyvale, CA, USA).

\section{Analysis of cDNA array data}

The current authors compared gene expression patterns of fibroblasts from the three groups after incubation with supernatant of 16HBE cells, CD8 cells, or the combination and at basal culture conditions, using AtlasImage 2.0 software (BD Biosciences Clontech). In order to subtract the common variance and find the differentially expressed genes, a new method was designed based on the data reduction tool "principal component analysis" [22-24].

Briefly, a matrix was prepared with all filter data placed in columns. To each value, 1 was added to avoid values of zero. The natural logarithm was taken to improve normality according to the following formula:

$$
\mathrm{A}^{\prime}=1.442695 \times \ln (\mathrm{A}+1)
$$

All columns were standardised to mean $=0$ and $\mathrm{SD}=1$. Subsequently, the principal components were calculated and the first principal component was deducted from the data matrix. Of the remaining data, the inverse of the natural logarithm was taken. Ratios were determined and a gene expression ratio of at least a two-fold increase compared with basal culture conditions was considered relevant.

\section{Real time PCR}

To obtain a more uniform patient group the only patients who had longstanding bronchitis (COPD stage IV) were excluded for PCR and subsequently for the soluble intercellular adhesion molecule (sICAM) ELISA and immunohistochemistry. To validate the results of the array data, quantitative real time PCR analysis was performed for ICAM-1 mRNA expression on individual patient fibroblast samples used in the array study. A total of $3 \mathrm{ng}$ of mRNA was transcribed into cDNA by reverse transcriptase II (Invitrogen, Breda, the Netherlands). Real time PCR was performed on an ABI7900HT sequencer with Assay on Demands from Applied Biosystems (Foster City, CA, USA), according to manufacturer's instructions. Data were analysed by the $\Delta \Delta \mathrm{CT}$ method [25]. In brief, CT values of the genes of interest were corrected for CT values from a household gene (ribosomal protein S9), resulting in a $\Delta \mathrm{CT}$ value. This $\Delta \mathrm{CT}$ was normalised to the average $\Delta \mathrm{CT}$ value of the basal control group. The log value was taken and ratios between stimulated and basal condition were calculated. Medians were taken from the individual patients per category.

\section{sICAM protein expression}

Of all fibroblasts samples, supernatants were harvested to determine sICAM levels produced by fibroblasts. sICAM levels were determined by a commercial sICAM ELISA kit (CLB/Sanquin, Amsterdam, the Netherlands). In brief, wells 
were coated with a capturing sICAM antibody and subsequently incubated with the supernatant samples. After incubation, wells were washed and incubated with a peroxidase labelled anti-sICAM antibody. Staining was visualised using 3,3',5,5'-tetramethylbenzidine. Absorption was read at $450 \mathrm{~nm}$ on a standard photospectrometer.

\section{Immunohistochemistry}

Immunohistochemistry was performed on $3 \mu \mathrm{m}$ formalin fixed, paraffin embedded lung tissue using a standard twostep immunoperoxidase protocol. Sections were incubated with a primary monocolonal antibody recognising ICAM-1 (kind gift from A.W. Boyd, Royal Melbourne Hospital, Melbourne, Australia) [26]. Envision (Dakopatts, Glostrup, Denmark) in combination with 3,3'-diaminobenzidine (Sigma, St. Louis, MO, USA) was used to visualise antibody binding. Slides were semiquantitatively scored for the number of ICAM-1+ cells in the submucosa. (1: few ICAM-1+ cells, 2: moderate number of ICAM-1+ cells, 3: large number of ICAM$1+$ cells).

\section{Statistical analysis}

Differences in subject characteristics, real-time data and immunohistochemistry scores between the three study groups were analysed using the Kruskall-Wallis test followed by the Mann-Whitney U-test. Differences in smoking habits and sex between the three study groups were analysed using the Fisher's exact test. The level of significance used was 0.05. All reported p-values are two-sided.

\section{RESULTS}

\section{Patient groups}

Pack-yrs smoking histories were similar in subjects with moderate/severe and very severe COPD (table 1). Patients with very severe COPD were significantly younger than patients with moderate COPD. Material from the current study's stage II group mainly consisted of males, material of the stage IV group mainly consists of females. From the present authors'archives, it was not possible to obtain material that was equally divided in males and females per COPD stage. The control group did not significantly differ in age or sex distribution from the COPD groups. Lung material from disapproved donor lungs was used as a control group. It was not possible to obtain all clinical characteristics of the donors, as could be obtained form the current study's patient group. However, this material was analysed by an experienced pathologist and classified as normal tissue.

\section{Array results in general}

A total of 51 out of 265 genes tested were differentially expressed on the cell interaction arrays. Genes were categorised into three groups: genes involved in adhesion and migration, genes involved in ECM production or modulation and genes involved in cellular regulation. Overall, cellinteraction genes were especially differentially expressed in the control and stage IV group. The stage II/III group showed only minor changes in gene expression profiles.

\section{Gene expression with epithelium supernatant stimulation}

After epithelial supernatant stimulation, 22 out of 265 genes were differentially expressed when compared with fibroblasts under basal culture conditions (table 2); in particular genes involved in adhesion and regulation. Epithelial supernatant influenced predominantly gene expression in control fibroblasts, but had hardly any effect on COPD fibroblasts. One gene in particular was differentially expressed: ICAM-1 expression was downregulated (-4.4 times) in the control group and, in contrast, upregulated in both COPD groups (4.5and 5.7-fold in stage II/III and stage IV, respectively).

\section{Gene expression after CD8 supernatant stimulation}

A total of 29 genes were differentially expressed upon CD8 supernatant stimulation (table 2), with 23 genes being differentially expressed in the GOLD stage IV COPD patient group and only nine and three genes expressed in GOLD stage II/III and control groups, respectively. Most regulated genes reflected adhesion processes and ECM substances. Notably ICAM-1 expression was highly upregulated in both COPD groups (10.2- and 9.5-fold in GOLD stage II/III and GOLD stage IV, respectively) whereas the control group showed no regulation of ICAM-1 mRNA expression.

\section{Gene expression after combined CD8 and epithelial supernatant}

After stimulation with a combination of CD8 and epithelial supernatants, 24 genes were differentially regulated (table 2). Fibroblasts of controls treated with the combined supernatants primarily showed alteration in the expression of genes involved in cell regulatory processes. Fibroblasts of GOLD stage II/III COPD patients showed minimal alteration under these conditions whereas in the GOLD stage IV COPD group, ICAM-1 showed a surprisingly high increase in mRNA expression (25.5-fold).

\section{Real time PCR}

Arrays were used to find indications for which genes would be most fit for further analysis of genes of interest by real time PCR. ICAM-1 expression was the most prominent change found in the arrays to explore and confirm by RT-PCR. The current authors found the highest ICAM-1 expression in GOLD stage IV fibroblasts under the influence of the epithelial and CD8 supernatant combination (fig. 1). Epithelial supernatant alone also significantly elevated the ICAM-1 gene expression in GOLD stage IV compared with GOLD stage II and controls. The CD8 supernatants also caused the highest level of ICAM-1 mRNA expression in the GOLD stage IV group, but this was not significant, due to large patient variation in combination with the relatively small number of subjects.

\section{sICAM protein expression}

To verify the mRNA expression, sICAM levels in the supernatants of all fibroblast samples were also determined, using an sICAM ELISA. Very small concentrations of sICAM were detected under basal conditions. Stimulation with the combination of CD8 and epithelial supernatant showed a trend towards an increase in sICAM levels in GOLD stage IV fibroblasts ( $p=0.055$, fig. 2). Epithelial and CD8 supernatant stimulation alone did not affect the protein levels of sICAM. 
TABLE 2 Gene expression of adhesion-related gene fibroblasts stimulated with supernatants of epithelial, CD8 cells or the combination compared with basal culture conditions

\begin{tabular}{|c|c|c|c|c|c|c|c|c|c|}
\hline Genes & \multicolumn{3}{|c|}{ Epithelial } & \multicolumn{3}{|c|}{ CD8 } & \multicolumn{3}{|c|}{ Epithelial and CD8 } \\
\hline \multicolumn{10}{|l|}{ Adhesion-related genes } \\
\hline CD27L & & & & 4.5 & & 8.1 & & & \\
\hline ICAM-1 & -4.4 & 4.5 & 5.7 & & 10.2 & 9.5 & 3.9 & & 25.5 \\
\hline Envoplakin & & & 2.2 & 3.4 & & 3.3 & & & 2.1 \\
\hline CD47 & 2.1 & & & 3.0 & & 4.9 & & & \\
\hline Ninjurin-1 & & 2.5 & & & & 2.2 & -2.0 & & 3.2 \\
\hline Ezrin & & & & 3.2 & & 8.0 & 2.4 & & \\
\hline Desmocollin & & & & -2.5 & & & -2.5 & & \\
\hline$\beta$ catenin & 2.4 & & & & & & & & \\
\hline Desmoplakin III & 2.2 & & & 2.4 & & & & & \\
\hline Integrin- $\alpha 8$ & & & & & & 2.9 & 2.0 & & \\
\hline VNR- $\alpha$ & 2.3 & & & & & & & & \\
\hline CD36 antigen & -2.2 & & & & & & & & \\
\hline Semaphorin; CD100 & -2.2 & & & & & & & & \\
\hline Semaphorin E & & 2.0 & & & & & & & \\
\hline CD9 antigen, MRP-1 & & & & & -2.1 & & & & \\
\hline E-selectin & -2.4 & & & & & & & & 2.0 \\
\hline CD153 antigen & -2.2 & & & & & & & & \\
\hline \multicolumn{10}{|l|}{ Matrix-related genes } \\
\hline FN precursor & & & & & & 2.4 & & & 2.3 \\
\hline SPARC & & & & & & -2.7 & & & \\
\hline Decorin & & & & & & 2.3 & & & \\
\hline \multicolumn{10}{|l|}{ Regulation-related genes } \\
\hline FAS-L & -3.6 & & & -2.3 & & & & & \\
\hline ILGF-BP 6 & & & & & & & -2.2 & & \\
\hline MARCKS-related protein & 2.5 & & & 2.0 & & 2.3 & 2.0 & & \\
\hline Ras-like protein TC10 & & & 2.1 & & & & & & \\
\hline Ras-related $\mathrm{C} 3$ botulinum toxin & 2.2 & & & & & 3.0 & -2.0 & & 2.5 \\
\hline Transforming protein rhoB & & & & & -2.2 & & & & \\
\hline TIAM-1 & & & 3.5 & & & & & 2.9 & \\
\hline Thrombospondin 1 & & & & 2.2 & & & 2.0 & & \\
\hline$\alpha_{2}$-Macroglobulin & & & & & & 2.3 & & & \\
\hline CD91 & & & & & & -2.3 & 2.1 & & \\
\hline $\mathrm{CD} 40 \mathrm{~L}$ & & & & & & & 2.1 & & 2.3 \\
\hline CD59 & & & & & & & 2.1 & & \\
\hline Lymphotoxin- $\alpha$ & -2.9 & & & & & & & & \\
\hline TNFR & & & & & & & 2.1 & & \\
\hline Neurogenic locus notch protein & & & & & & & -2.1 & & \\
\hline DLK & -2.2 & & & & & & & & \\
\hline
\end{tabular}




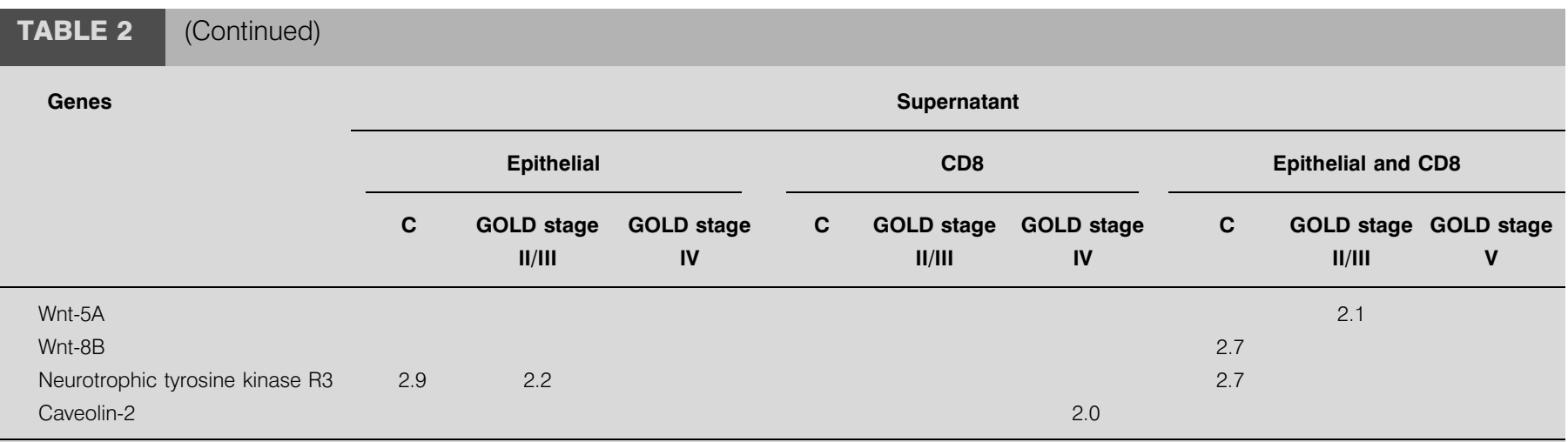

C: control group; GOLD: Global Initiative for Chronic Obstructive Lung Disease (GOLD); LFA-1; Iymphocyte function-associated antigen-1; ICAM-1: intercellular adhesion molecule-1; VNR- $\alpha$ : vitronectin receptor- $\alpha$; MRP-1: motility-related protein-1; TIMP-1; tissue inhibitor of metalloproteinases-1; FN: fibronectin precursor; SPARC: secreted protein acidic and rich in cysteine; FAS-L: FAS ligand; ILGF-BP-6: insulin-like growth factor binding protein 6; MARCKS: myristoylated alanine-rich protein C kinase substrate; TIAM-1; T-cell lymphoma invasion and metastasis-1; TNFR: tumour necrosis factor receptor; DLK: delta-like protein precursor. Ratios were determined between stimulated fibroblasts and fibroblasts under basal culture conditions. A minimum of two-fold higher or lower expression is considered relevant.

\section{Immunohistochemistry}

To further verify the highly elevated mRNA expression of GOLD stage IV fibroblasts, ICAM-1 protein expression in tissue sections of the same control and COPD patients from whom fibroblasts were derived, as used in the array study, were also analysed. In the submucosa, where a large number of fibroblasts are located and is likely to be influenced by local epithelial and CD8-derived factors, a significantly larger number of ICAM-1+ cells were found in GOLD stage IV COPD patients when compared with controls and GOLD stage II/III COPD patients (fig. 3). Most positive cells represented elongated fibroblast-like cells (fig. 4). This supports the present authors' observation of the high increase in ICAM-1 mRNA expression in GOLD stage IV COPD fibroblasts.

\section{DISCUSSION}

Smoking is known to induce CD8+ T-cell influx and epithelial activation in the airway wall of COPD patients. The present authors explored the modulating effects of CD8+ T-cells and epithelial cell-derived mediators on gene expression profiles of fibroblasts obtained from GOLD stages II/III, and IV, as well as controls. The current authors clearly established that the cytokine microenvironment differentially modulates pulmonary fibroblast gene expression in COPD patients compared with the controls. In particular adhesion-related genes were differentially expressed and an interaction was apparent when the fibroblasts were exposed to the combination of the CD8 and epithelial supernatants. Thus, factors in the microenvironment of the fibroblast may contribute to the altered fibroblast behaviour observed in COPD. Since fibroblasts are essential cells in the modulation of the ECM, an alteration in their behaviour could reflect the underlying cause of the defective ECM repair observed in COPD.

The current experiments were performed as closely related to the actual lung microenvironment as possible, but they still represent an in vitro model for the in vivo situation. The use of supernatants represents a source of uncertainty and variation but the alternative, to use combinations of individual cytokines, would have resulted in a selection bias. CD8+ T-cells were used from peripheral blood of COPD patients as a representative for the CD8 T-cells in the diseased lung. A primary epithelial cell line was used as it is not possible to obtain sufficient cultures of primary epithelial cells and fibroblasts from the same patient. The current model is an appropriate model reflecting the microenvironment in COPD in order to study the role of mediators derived from CD8 Tcells and epithelial cells on fibroblasts.

ICAM-1 showed a striking differential gene expression pattern between COPD and controls, which was supported by elevated production of SICAM in combination with elevated expression in lung tissue of COPD patients. In the arrays, the combination of epithelial and CD8 supernatant showed the largest differential expression in GOLD stage IV fibroblasts. This observation was confirmed by results from the real time PCR and was also supported by finding a near-significant trend $(p=0.055)$ towards a higher production of sICAM-1 by GOLD stage IV fibroblasts as compared with controls and GOLD stage II/III fibroblasts. In addition, GOLD stage IV COPD patients showed the largest number of ICAM-1+ cells $(p=0.0079)$, mostly fibroblasts, in the submucosa of the small airways where an influence of CD8 cells, as well as epithelial cells, is to be expected. Although an effect of supernatant of epithelial cells and of CD8 cells on ICAM gene expression seemed to be present in the macroarray, this could not be confirmed using RT-PCR.

ICAM-1 is an important adhesion molecule involved in a number of inflammatory processes, such as neutrophil and lymphocyte trafficking and it acts as an accessory molecule in antigen presentation [27]. ICAM-1 expression is regulated by a variety of cytokines, e.g. interleukin (IL)-1, IL-4, interferon gamma, TGF- $\beta$ and tumour necrosis factor (TNF)- $\alpha[27,28]$. The CD8+ T-cells known to be elevated in number in COPD patients are an important source of IL- 4 and TNF- $\alpha$, while epithelial cells are an important source of TGF- $\beta$. Thus these cytokines may be the key element of the supernatants explaining the elevated ICAM-1 expression.

Several studies have pointed to a role of ICAM-1 expression in the pathogenesis of COPD. ICAM-1 concentrations in bronchoalveolar lavage of COPD patients are increased and cigarette 

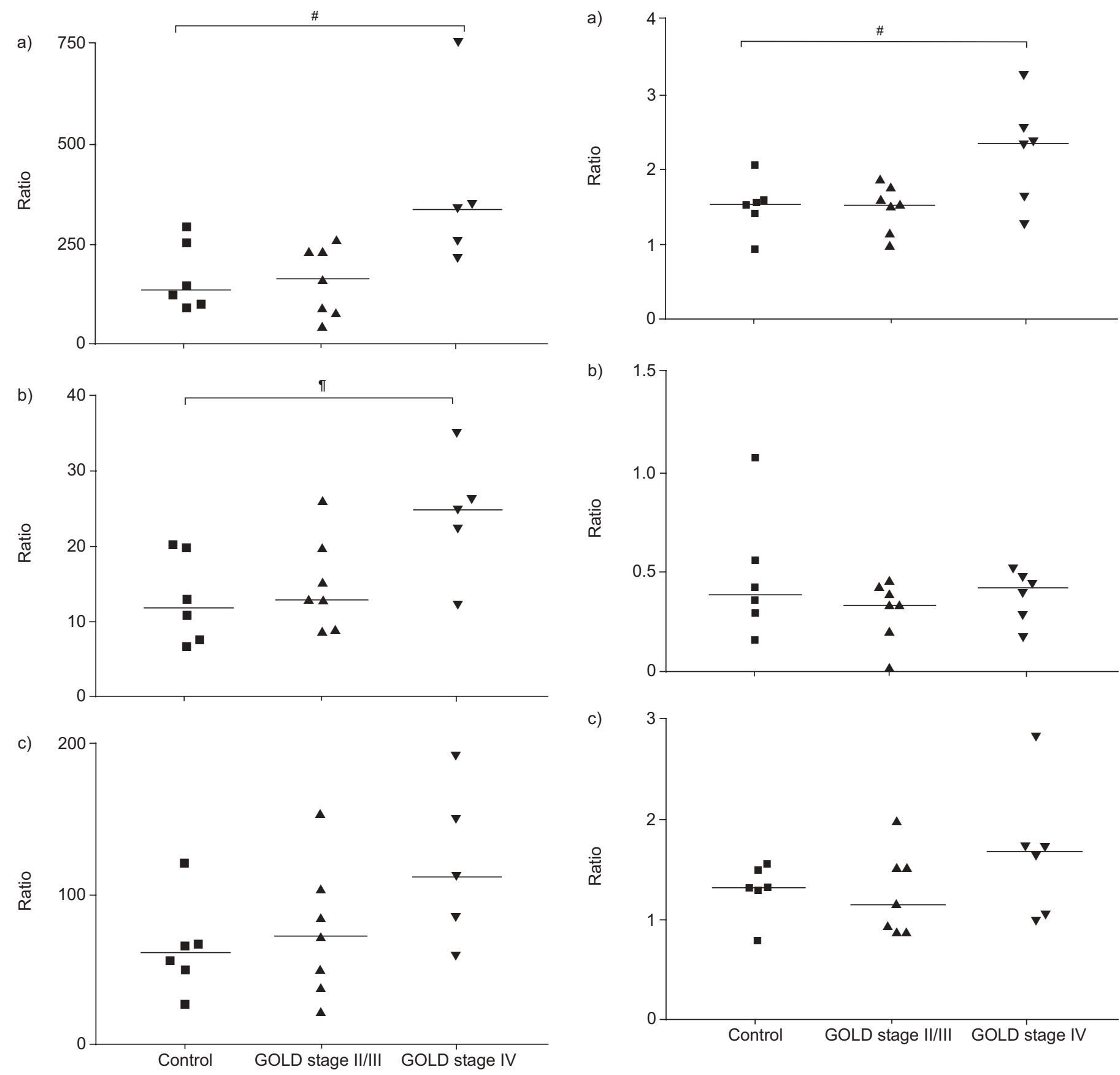

FIGURE 1. Real time intercellular adhesion molecule-1 mRNA expression of fibroblasts stimulated with supernatants of a) epithelial and CD8 cells, b) epithelial cells or c) CD8 cells. Ratio compared with basal culture conditions. GOLD: Global Initiative for Chronic Obstructive Lung Disease. -: median. \#: $p=-0.028$; ": $p=-0.028$. One GOLD stage IV sample was lost due to technical reasons.

smoke extract increases ICAM-1 production by macrophages $[29,30]$. In addition, an impressive decline in plasma ICAM-1 concentration occurred after smoking cessation [31]. Finally, fibroblasts are able to interact with inflammatory cells via adhesion molecules, such as ICAM-1 and vascular cell adhesion molecule-1 [28], and increased ICAM-1 expression is associated with leukocyte transmigration along fibroblasts [32]. Therefore, it is likely that an increased ICAM-1 expression

FIGURE 2. Soluble intercellular adhesion molecule-1 protein expression of fibroblasts stimulated with supernatants of a) epithelial and CD8 cells, b) epithelial cells or c) CD8 cells. Ratio compared with basal culture conditions. GOLD: Global Initiative for Chronic Obstructive Lung Disease. ${ }^{*}: p=0.055$.

on fibroblasts of COPD patients points to important interactions with inflammatory cells. It is known that increased numbers of CD8+ T-lymphocytes are present throughout the lung in smoking subjects with COPD [13, 16, 33-35], whereas epithelial cells cover more centrally located airways. The cytokines produced by both CD8+ T-cells and epithelial cells are known modulators of fibroblast activity in COPD $[2,13,36$, 37]. The present authors used supernatant of stimulated CD8+ T-cells and epithelial cells to create an in vitro model reflecting the in vivo situation of pulmonary fibroblasts. An important 


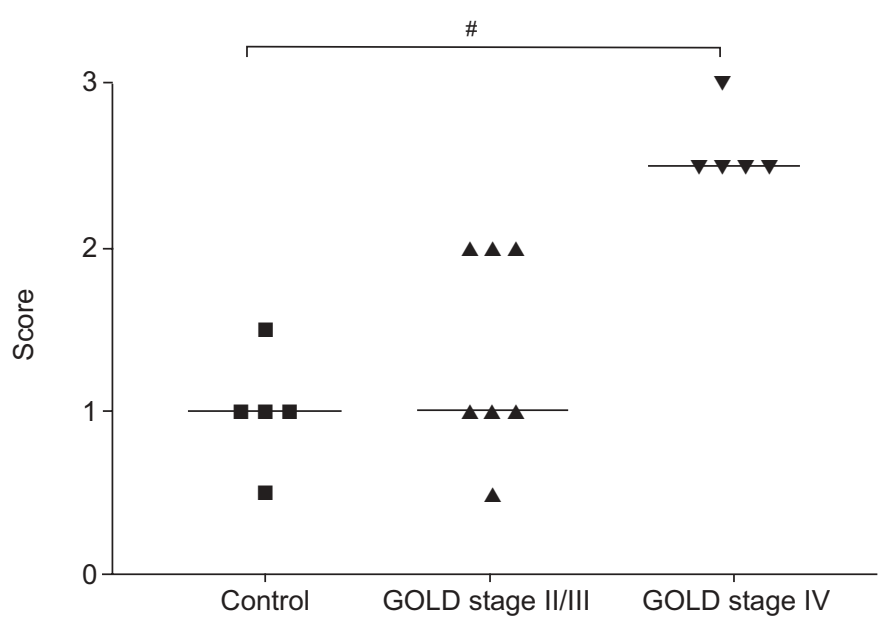

FIGURE 3. Semi-quantitative score of number of intercellular adhesion molecule (ICAM-1)+ cells in the submucosa. Relative frequency of ICAM-1+ cells is significantly increased in Global Initiative for Chronic Obstructive Lung Disease (GOLD) stage IV chronic obstructive pulmonary disease.

aspect of the current study is that a supernatant of CD8+ Tcells, isolated from peripheral blood of COPD patients without chronic bronchitis, was used, as CD8+ T-cells of COPD patients have been shown to possess an altered cytokine production profile [15, 35]. Thus, more centrally (bronchially) located fibroblasts are modulated by both epithelial and CD8-derived factors, whereas more distally (parenchymal) located fibroblasts will be less regulated by bronchial epithelial cell-derived factors. In the specific microenvironments of the bronchial area, where both sources of mediators are present, the balance between mediators of epithelial and CD8+ T-cells may ultimately determine the effect on fibroblast gene expression in COPD patients.

Fibroblasts are multipotent cells, their activity and function can be modulated based on local environmental factors. A major feature of fibroblasts is the production of ECM components, initiated in part by TGF- $\beta$ stimulation. Fibroblasts are also involved in immunological processes, playing an important role in the switch from an acute, resolving inflammation to a chronic persistent inflammation [17, 18]. BUCKLEY et al. [18] suggested that this chronic inflammation occurs because of disordered fibroblast behaviour, which leads to inappropriate survival and retention of leukocytes within inflamed tissue [38]. Thus it is not surprising that fibroblasts in COPD tissue show a functionally altered behaviour under the influence of the local cytokine microenvironment $[11,18]$. The differentially expressed adhesion-related genes found in GOLD stage IV COPD fibroblasts when stimulated with CD8 supernatant supports the theory of a prominent role of fibroblasts in the chronic inflammatory process observed in COPD.

Besides the strong upregulation of ICAM-1, several other adhesion-related genes were shown to be upregulated. It seems likely, therefore, that COPD fibroblasts, especially in GOLD stage IV COPD, are easily induced by the local cytokine microenvironment to interact with inflammatory cells and thus play a supportive role in the ongoing inflammation. Fibroblasts differentiated to a more immunological orientated function may not be fully capable of maintaining the ECM or may exhibit altered ECM repair mechanisms. These functional changes could contribute to destruction or inadequate maintenance of the ECM, causing some of the specific features of COPD. The current authors have previously demonstrated a decreased decorin and biglycan immunohistological staining in lung tissue of GOLD stage IV COPD patients, signifying the altered ECM modulation [10].

In conclusion, fibroblasts of chronic obstructive pulmonary disease patients display an altered response to the cytokine microenvironment in the chronic obstructive pulmonary diseased lung, depending on both the disease stage and the central or peripheral location in the lung. The local cytokine micro-environment appears to skew the chronic obstructive pulmonary disease fibroblasts to interact with inflammatory cells instead of a focus on extracellular matrix maintenance, which may be pivotal in the pathogenesis of chronic obstructive pulmonary disease. Furthermore the current findings suggest that tissue repair mechanisms will differ in the parenchyma versus the bronchial wall in chronic obstructive pulmonary disease.
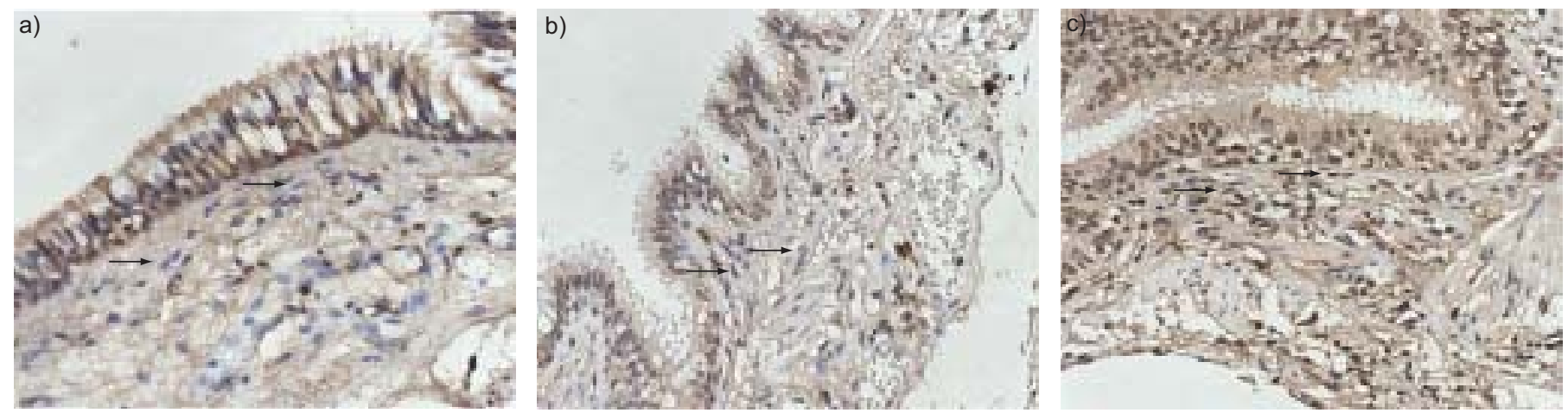

FIGURE 4. Intercellular adhesion molecule (ICAM)-1 staining (brown) in lung tissue of a) control, b) Global Initiative for Chronic Obstructive Lung Disease (GOLD) stage II COPD and c) GOLD stage IV COPD subjects. In the control, as well as GOLD stage II COPD, very small numbers of ICAM-1+ cells were observed in the submucosa. Arrows indicate elongated ICAM-1 negative fibroblasts in the submucosa. In GOLD stage IV COPD, numerous ICAM-1+ cells were observed. Arrows indicate the ICAM-1+ elongated fibroblasts. Cells were counterstained with haematoxylin. 


\section{ACKNOWLEDGEMENTS}

The authors would like to thank T.R. Bai for carefully reading the manuscript, G. te Meerman for designing the principal component analysis tool, D.C. Gruenert for kindly providing the 16HBE bronchial epithelial cell line and J. Liesker for providing the blood samples from well characterised chronic obstructive pulmonary disease patients.

\section{REFERENCES}

1 American Thoracic Society. Definitions, epidemiology, pathophysiology, diagnosis, and staging. Am J Respir Crit Care Med 1995; 152: S78-S83.

2 Turato G, Zuin R, Saetta M. Pathogenesis and pathology of COPD. Respiration 2001; 68: 117-128.

3 Barnes PJ. New concepts in chronic obstructive pulmonary disease. Annu Rev Med 2003; 54: 113-129.

4 Pauwels RA, Buist AS, Calverley PM, Jenkins CR, Hurd SS. Global strategy for the diagnosis, management, and prevention of chronic obstructive pulmonary disease. NHLBI/WHO Global Initiative for Chronic Obstructive Lung Disease (GOLD) Workshop summary. Am J Respir Crit Care Med 2001; 163: 1256-1276.

5 Calverley PM, Walker P. Chronic obstructive pulmonary disease. Lancet 2003; 362: 1053-1061.

6 Agusti A, MacNee W, Donaldson K, Cosio M. Hypothesis: does COPD have an autoimmune component? Thorax 2003; 58: 832-834.

7 Timens W, Coers W, van Straaten JFM, Postma DS. Extracellular matrix and inflammation: a role for fibroblast-mediated defective tissue repair in the pathogenesis of emphysema? Eur Respir Rev 1997; 7: 119-123.

8 van der Geld YM, van Straaten JF, Postma DS, Timens W. Role of proteoglycans in the development and pathogenesis of emphysema. In: Garg HG, Roughley PJ, Hales CA, eds. Proteoglycans in Lung Disease. New York, Marcel Dekker Inc., 2002; pp. 241-267.

9 Dunsmore SE, Rannels DE. Extracellular matrix biology in the lung. Am J Physiol 1996; 270: L3-L27.

10 van Straaten JF, Coers W, Noordhoek JA, et al. Proteoglycan changes in the extracellular matrix of lung tissue from patients with pulmonary emphysema. Mod Pathol 1999; 12: 697-705.

11 Noordhoek JA, Postma DS, Chong LL, et al. Different proliferative capacity of lung fibroblasts obtained from control subjects and patients with emphysema. Exp Lung Res 2003; 29: 291-302.

12 Noordhoek JA, Postma DS, Chong LL, et al. Different modulation of decorin production by lung fibroblasts from patients with mild and severe emphysema. J COPD 2005; 2: 17-25.

13 Cosio MG, Majo J, Cosio MG. Inflammation of the airways and lung parenchyma in COPD: role of T cells. Chest 2002; 121: Suppl. 5, 160S-165S.

14 Reid PT, Sallenave JM. Cytokines in the pathogenesis of chronic obstructive pulmonary disease. Curr Pharm Des 2003; 9: 25-38.

15 Mattoli S, Kleimberg J, Stacey MA, Bellini A, Sun G, Marini M. The role of CD8+ Th2 lymphocytes in the development of smoking-related lung damage. Biochem Biophys Res Commun 1997; 239: 146-149.
16 Barnes PJ, Shapiro SD, Pauwels RA. Chronic obstructive pulmonary disease: molecular and cellular mechanisms. Eur Respir J 2003; 22: 672-688.

17 Davis LS. A question of transformation: the synovial fibroblast in rheumatoid arthritis. Am J Pathol 2003; 162 : 1399-1402.

18 Buckley CD, Pilling D, Lord JM, Akbar AN, ScheelToellner D, Salmon M. Fibroblasts regulate the switch from acute resolving to chronic persistent inflammation. Trends Immunol 2001; 22: 199-204.

19 Barnes PJ. Mediators of chronic obstructive pulmonary disease. Pharmacol Rev 2004; 56: 515-548.

20 National Institute of Health, National Heart Lung and Blood Institute. Global strategy for the diagnosis, management, and prevention of chronic obstructive disease. Based on 1998 NHLBI/WHO workshop, April 2001. www.goldcopd. org/GuidelinesResources.asp? $11=2 \& 12=0$. Date last updated: 2003.

21 Haws C, Krouse ME, Xia Y, Gruenert DC, Wine JJ. CFTR channels in immortalized human airway cells. Am J Physiol 1992; 263: L692-L707.

22 Quackenbush J. Microarray data normalization and transformation. Nat Genet 2002; 32: Suppl., 496-501.

23 Simon R, Radmacher MD, Dobbin K, McShane LM. Pitfalls in the use of DNA microarray data for diagnostic and prognostic classification. J Natl Cancer Inst 2003; 95: 14-18.

24 Liu A, Zhang Y, Gehan E, Clarke R. Block principal component analysis with application to gene microarray data classification. Stat Med 2002; 21: 3465-3474.

25 Livak KJ, Schmittgen TD. Analysis of relative gene expression data using real-time quantitative PCR and the 2(-Delta Delta C(T)) Method. Methods 2001; 25: 402-408.

26 Boyd AW, Wawryk SO, Burns GF, Fecondo JV. Intercellular adhesion molecule 1 (ICAM-1) has a central role in cell-cell contact-mediated immune mechanisms. Proc Natl Acad Sci USA 1988; 85: 3095-3099.

27 Hubbard AK, Rothlein R. Intercellular adhesion molecule1 (ICAM-1) expression and cell signaling cascades. Free Radic Biol Med 2000; 28: 1379-1386.

28 Sabatini F, Silvestri M, Sale R, et al. Fibroblast-eosinophil interaction. Modulation of adhesion molecules expression and chemokine release by human fetal lung fibroblasts in response to IL-4 and TNF-alpha. Immunol Lett 2002; 84: 173-178.

29 Riise GC, Larsson S, Lofdahl CG, Andersson BA. Circulating cell adhesion molecules in bronchial lavage and serum in COPD patients with chronic bronchitis. Eur Respir J 1994; 7: 1673-1677.

30 Zhang $\mathrm{X}$, Wang L, Zhang $\mathrm{H}$, et al. The effects of cigarette smoke extract on the endothelial production of soluble intercellular adhesion molecule- 1 are mediated through macrophages, possibly by inducing TNF-alpha release. Methods Find Exp Clin Pharmacol 2002; 24: 261-265.

31 Scott DA, Stapleton JA, Wilson RF, et al. Dramatic decline in circulating intercellular adhesion molecule-1 concentration on quitting tobacco smoking. Blood Cells Mol Dis 2000; 26: 255-258.

32 Tessier PA, Cattaruzzi P, McColl SR. Inhibition of lymphocyte adhesion to cytokine-activated synovial fibroblasts by glucocorticoids involves the attenuation of vascular cell adhesion molecule 1 and intercellular 
adhesion molecule 1 gene expression. Arthritis Rheum 1996; 39: 226-234.

33 Saetta M, Di Stefano A, Turato G, et al. CD8+ Tlymphocytes in peripheral airways of smokers with chronic obstructive pulmonary disease. Am J Respir Crit Care Med 1998; 157: 822-826.

34 Hogg JC, Chu F, Utokaparch S, et al. The nature of smallairway obstruction in chronic obstructive pulmonary disease. N Engl J Med 2004; 350: 2645-2653.

35 Barnes PJ, Cosio MG. Characterization of T lymphocytes in chronic obstructive pulmonary disease. PLoS Med 2004; 1: e20.
36 Doucet C, Brouty-Boye D, Pottin-Clemenceau C, Canonica GW, Jasmin C, Azzarone B. Interleukin (IL) 4 and IL-13 act on human lung fibroblasts. Implication in asthma. J Clin Invest 1998; 101: 2129-2139.

37 Spoelstra FM, Postma DS, Hovenga H, Noordhoek JA, Kauffman HF. Interferon-gamma and interleukin-4 differentially regulate ICAM-1 and VCAM-1 expression on human lung fibroblasts. Eur Respir J 1999; 14: 759-766.

38 Douglas MR, Morrison KE, Salmon M, Buckley CD. Why does inflammation persist: a dominant role for the stromal microenvironment? Expert Rev Mol Med 2002; 9: 1-18. 